\title{
Charged defects during alpha-irradiation of actinide oxides as revealed by Raman and luminescence spectroscopy
}

\author{
Mohun $\mathrm{R}^{\mathrm{a}}$, Desgranges $\mathrm{L}^{\mathrm{a}}$, Simon $\mathrm{P}^{\mathrm{b}}$, Guimbretière $\mathrm{G}^{\mathrm{b}}$, Canizarès $\mathrm{A}^{\mathrm{b}}$, Duval $\mathrm{F}^{\mathrm{b}}$, Jegou $\mathrm{C}^{\mathrm{c}}$, Magnin $\mathrm{M}^{\mathrm{C}}$, Clavier \\ $\mathrm{N}^{\mathrm{d}}$, Dacheux $\mathrm{N}^{\mathrm{d}}$ \\ a. CEA, DEN, DEC/SESC, Centre de Cadarache, 13108 Saint-Paul-lez-Durance \\ b. CNRS, UPR 3079 CEMHTI, et Université d'Orléans, 1D avenue de la Recherche Scientifique, 45071 Orléans \\ c. CEA, DEN, DTCD, Centre de Marcoule, BP 17171, 30207 Bagnols sur Cèze \\ d. ICSM, UMR 5257 CEA/CNRS/UM2/ENSCM, Site de Marcoule, BP 17171, 30207 Bagnols sur Cèze
}

\begin{abstract}
We have recently evidenced an original Raman signature of alpha irradiation-induced defects in $\mathrm{UO}_{2}$. In this study, we aim to determine whether the same signature also exists in different actinide oxides, namely $\mathrm{ThO}_{2}$ and $\mathrm{PuO}_{2}$. Sintered $\mathrm{UO}_{2}$ and $\mathrm{ThO}_{2}$ were initially irradiated with $21 \mathrm{MeV} \mathrm{He}{ }^{2+}$ ions using a cyclotron device and were subjected to an in situ luminescence experiment followed by Raman analysis. In addition, $\mathrm{a} \mathrm{PuO}_{2}$ sample that has previously accumulated self-irradiation damage due to alpha particles was investigated only by Raman measurement. Results obtained for the initially white $\mathrm{ThO}_{2}$ showed that a blue color appeared in the irradiated areas as well as luminescence signals during irradiation. However, Raman spectroscopic analysis showed the absence of Raman signature in $\mathrm{ThO}_{2}$. In contrast, the irradiated $\mathrm{UO}_{2}$ and $\mathrm{PuO}_{2}$ confirmed the presence of the Raman signature but no luminescence peaks were observed. These findings lead to propose a mechanism involving electronic defects in $\mathrm{ThO}_{2}$ while a coupling between electronic defects and phonons is needed to explain the Raman signal in $\mathrm{UO}_{2}$ and $\mathrm{PuO}_{2}$.
\end{abstract}

Keywords: Alpha irradiation; Raman signature; Luminescence; Electronic defects

1. Introduction

Accurate determination of nuclear fuel behaviors in normal or accidental condition is a challenge for the nuclear industry in order to ensure its safety and profitability. Irradiation damages have a direct impact on the physical properties of the nuclear fuels and several theoretical works are currently in progress aiming to determine the stable point defect in $\mathrm{UO}_{2}$. Unfortunately, limited experimental data is available to characterize irradiation defects in nuclear fuels. X-Ray diffraction and Transmission Electron Microscope evidenced changes in the crystalline structure and grain microstructure during irradiation [1-2]; however these methods are not well suited to evidence point defects. Recently [3] evidenced a specific signature of irradiation in $\mathrm{UO}_{2}$ using the Raman spectroscopy method. This signal corresponds to three additional peaks in the Raman spectrum of $\mathrm{UO}_{2}$ implanted with alpha ions and can be related to a single defect that is annealed between 300 and $525{ }^{\circ} \mathrm{C}$ [4]. According to [5] its creation kinetics can be modelled by a single impact model and has previously been used to describe $\mathrm{UO}_{2}$ cell parameter behavior under irradiation. These results observed by Raman spectroscopy has characteristics similar to irradiation-induced point defects, and therefore deserved an in-depth characterization and understanding.

A striking feature of the Raman defect arises from its creation process. According to SRIM simulation code, a $25 \mathrm{MeV}$ Helium ion beam will primarily lose its energy through electronic stopping. However, the electronic energy due to the alpha ions $(\mathrm{dE} / \mathrm{dx}=1 \mathrm{keV} / \mathrm{nm})$ is much weaker that the value reported in literature for creation of tracks by swift heavy ions [6]. In that range of deposited energy, only self-trapped excitons or colored defects could be formed [7]. To date the existence of such defect types has not been reported about irradiated $\mathrm{UO}_{2}$.

This paper is devoted to understand the behavior of three actinide oxides namely $\mathrm{UO}_{2}, \mathrm{ThO}_{2}$ and $\mathrm{PuO}_{2}$ since they all share the same crystallographic structure, with similar mass, density and number of electrons. The study aims to examine the existence of electronic processes in these oxides. Moreover the energy deposited profile in all these compounds, as determined by SRIM, shows same trend and this suggests that they should have similar behavior under irradiation.

2. Experimental Procedures

\subsection{Samples}

Sintered $\mathrm{UO}_{2}$ and $\mathrm{ThO}_{2}$ samples were prepared at CEA-Cadarache and ICSM respectively. $\mathrm{UO}_{2}$ discs were heat treated at $1700^{\circ} \mathrm{C}$ under dry $\mathrm{Ar} / \mathrm{H}_{2}$ while $\mathrm{ThO}_{2}$ was sintered at $1600^{\circ} \mathrm{C}$ in air. The discs were all mirror-polished at one side and then annealed at $1000^{\circ} \mathrm{C}$ to maintain stoichiometry and also remove polishing damages. In this work, the pellets were sectioned into two-half discs along their cylindrical axis as it allows to correlate the penetration profile of the ions along the depth of the samples with simulated data as described in [3]. The irradiation was performed at the Cyclotron facility, CEMHTI Orléans (France) and it was possible to carry out both in situ (Ion-Beam Induced Luminescence) and ex situ (Visual and Raman) experiments. 
$\mathrm{A} \mathrm{PuO}_{2}$ sample was initially prepared at CEA-Cadarache and heat treated at $1700^{\circ} \mathrm{C}$ under reducing environment for 2 hours back in 2011. The pellet showed a high alpha activity due to the presence of alpha emitters. To consider the effect of alpha ions, the $\mathrm{PuO}_{2}$ was left to decay for 2.5 years. The auto-irradiated pellet was later characterized with Raman spectroscopy in 2013.

\subsection{Irradiations}

The $\mathrm{UO}_{2}$ and $\mathrm{ThO}_{2}$ half-disks were all irradiated with $21 \mathrm{MeV} \mathrm{He}^{2+}$ during 2 hours and an ion flow of $50 \mathrm{nA}$ using the cyclotron device. The energy chosen was able to deliver more electronic energy $\left((\mathrm{dE} / \mathrm{dx})_{\mathrm{E}}=132 \mathrm{keV} / \mu \mathrm{m}\right)$ near the surface of the pellets. All irradiations were carried out at room temperature and under vacuum condition so as to prevent any risk of pellet oxidation.

\subsection{Ion-Beam Induced Luminescence Experiment (In situ)}

The luminescence set-up used to monitor the behavior of $\mathrm{UO}_{2}$ and $\mathrm{ThO}_{2}$ during irradiation consisted of an optical set-up of a pyrometer probe implemented on the CEMHTI cyclotron beam line and positioned in the direction of the pellet's surface exposed to the irradiation beam. An optical fiber coming from the pyrometer probe-head was connected to a one-grating monochromator (SR 163 Shamrock Andor) and an ICCD multichannel detector (Andor iStar DH720) all located in a safety room $20 \mathrm{~m}$ away from the irradiation cell, by means of optical fibers and electrical cables.

\subsection{Raman measurement (Ex situ)}

A Renishaw RA-100 Raman Analyzer was used to characterize fuel oxides before and after irradiation. A $633 \mathrm{~nm}$ He-Ne was initially employed and each Raman spectrum was acquired through an exposure time of 40s for a wavelength ranging from 300 to $700 \mathrm{~cm}^{-1}$. A second step consisted of analyzing the irradiated pellets using a $532 \mathrm{~nm}$ green laser so as to confirm the presence of Raman signals.

A similar procedure was applied for $\mathrm{PuO}_{2}$; however with a different experimental procedure. This set-up variance was necessary since the sample was within a glove box to prevent radiological contamination. Hence, the Raman head was placed outside the box and the laser was focused onto the sample passing through the glass of the glove compartment (Mitutoyo $\mathrm{x} 20$ objective, $30 \mathrm{~mm}$ front distance).

3. Results

\subsection{Ion-Beam Induced Luminescence}

The luminescence spectrum of $\mathrm{ThO}_{2}$ was recorded for a wavelength ranges from 200 to 800 nm during irradiation as shown in Fig. 1. Before irradiation $(\mathrm{t}=0 \mathrm{~min})$, the $\mathrm{ThO}_{2}$ sample emits no luminescence signals. The spectrum is largely modified given the number of peaks appearing during the initial stage of the $\mathrm{He}^{2+}$ beam. It can be seen that more emission occurs between 300 $\mathrm{nm}$ to $620 \mathrm{~nm}$ which could be related to the formation of electronic defects in the material as discussed by [8]. However under the same irradiation conditions, no luminescence peaks were observed in $\mathrm{UO}_{2}$ and the spectrum remained unchanged even when the ion flow was increased from $50 \mathrm{nA}$ up to $200 \mathrm{nA}$. This result provides evidence that a different defect mechanism is involved in $\mathrm{UO}_{2}$ compared to $\mathrm{ThO}_{2}$.

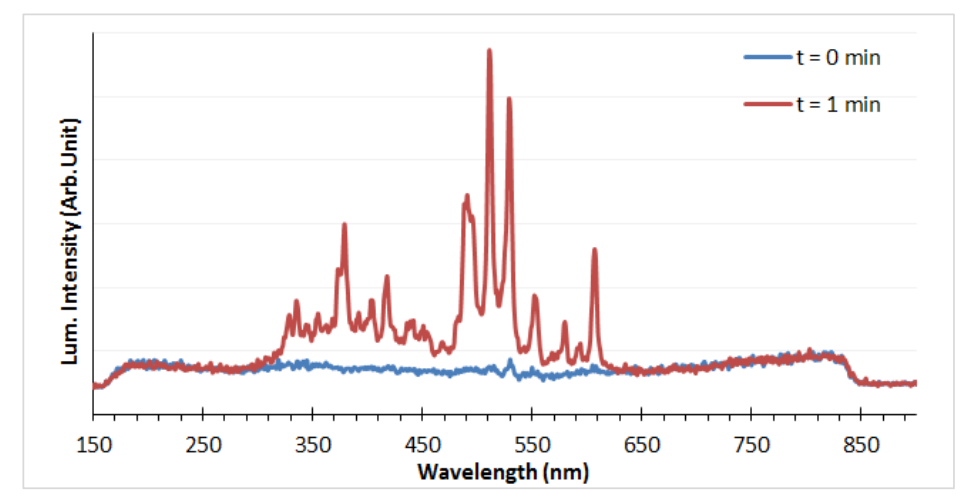

Fig. 1. Luminescence spectrum of $\mathrm{ThO}_{2}$ during $21 \mathrm{MeV} \mathrm{He}^{2+}$ and $50 \mathrm{nA}$ ion flow

\subsection{Visual Inspection}

The $\mathrm{UO}_{2}$ and $\mathrm{ThO}_{2}$ samples were both visually compared. Although $\mathrm{UO}_{2}$ exhibited no significant change in its physical appearance, the sections of the initially white $\mathrm{ThO}_{2}$ exposed to the irradiation beam turned into a deep blue color (Fig. 2). The coloration of $\mathrm{ThO}_{2}$ has previously been observed [9, 10, 11-12] and [13] reported that it take its origin from colored defects with swallow energy which can be annealed out at high temperature. 

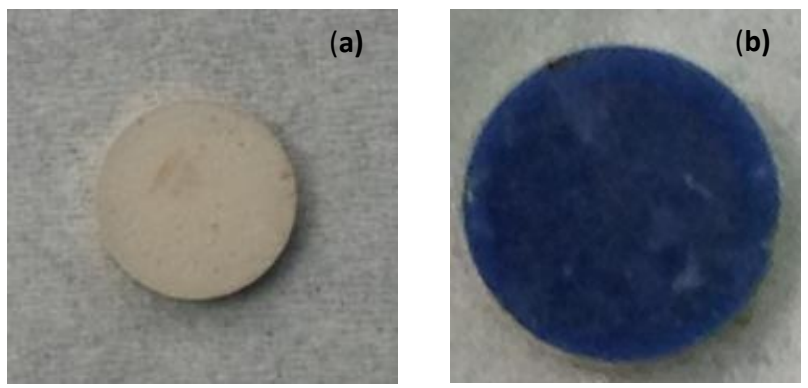

Fig. 2. $\mathrm{ThO}_{2}$ pellet (a) before and (b) after irradiation respectively

\subsection{Raman Spectroscopy}

\subsubsection{Before Irradiation}

As described in section 2.4, the $633 \mathrm{~nm}$ was initially used for the acquisition of the Raman spectra of the virgin pellets (Fig. 3). According to its phonon dispersion curves [14-15], $\mathrm{UO}_{2}$ has a single Raman active mode which corresponds to the symmetric $\mathrm{T}_{2 \mathrm{~g}}$ vibration mode occurring at $445 \mathrm{~cm}^{-1}$ [16-17]. The $\mathrm{T}_{2 \mathrm{~g}}$ peak is also signature of oxides exhibiting the fluorite structure sharing the Fm3m space group. Another peak with a small intensity appears at $575 \mathrm{~cm}^{-1}$ which is attributed to the LO phonon [18]. It is Raman forbidden in virgin $\mathrm{UO}_{2}$ and can be due to the presence of oxygen vacancies in the FCC structure [5]. ThO sharing the same fluorite structure as $\mathrm{UO}_{2}$, has its $\mathrm{T}_{2 \mathrm{~g}}$ peak occurring at $465 \mathrm{~cm}^{-1}$ as shown in Fig. 3(b).
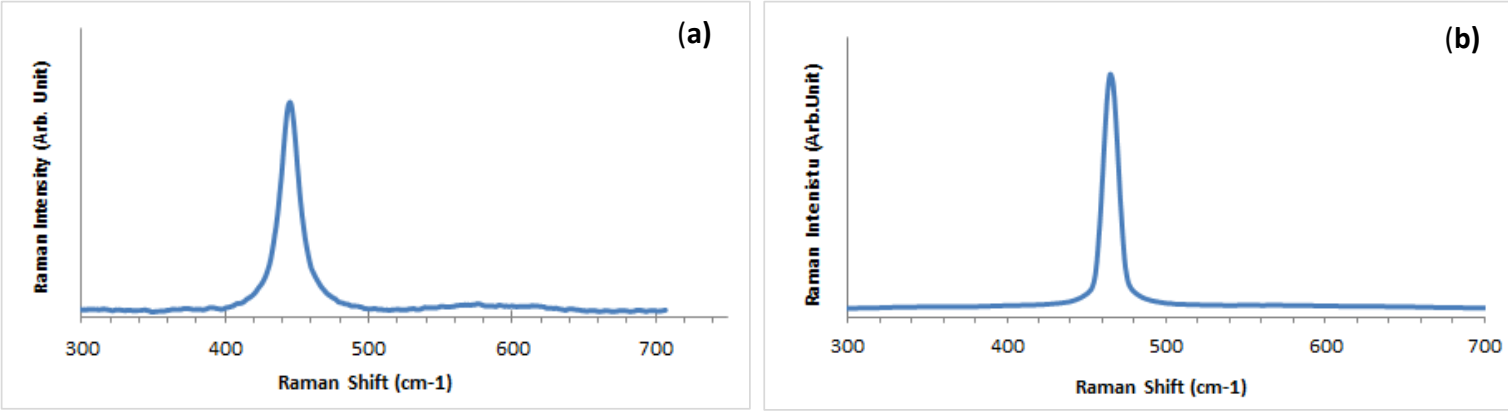

Fig. 3. Raman spectrum of (a) virgin $\mathrm{UO}_{2}$ and (b) $\mathrm{ThO}_{2}$ samples

Unfortunately, the initial Raman characterization of $\mathrm{PuO}_{2}$ just after the heat treatment could not be carried out. The structure of $\mathrm{PuO}_{2}$, identical to that of $\mathrm{UO}_{2}$, is a fluorite one with an $\mathrm{O}_{\mathrm{h}}$ space group and has previously been studied by Raman spectroscopy [19]. It has its $\mathrm{T}_{2 \mathrm{~g}}$ phonon peak occurring at $477 \mathrm{~cm}^{-1}[20]$.

\subsubsection{Post-Irradiation}

The Raman measurement was done on a cross-section of each irradiated half-disc at a distance of $106 \mu \mathrm{m}$ from the surface. This corresponds to the region where maximum electronic energy was deposited during irradiation. The results obtained from this characterization are described below.

\section{$\underline{\mathrm{UO}_{2}} \& \mathrm{PuO}_{2}$ Spectra}

Fig. 4(a) shows the Raman spectrum of irradiated $\mathrm{UO}_{2}$ when analyzed with the $633 \mathrm{~nm}$ laser. It can be seen that along with the $\mathrm{T}_{2 \mathrm{~g}}$, there are the presence of three additional peaks in the 500-700 $\mathrm{cm}^{-1}$ range denoted as $\mathrm{U}_{1}\left(\sim 527 \mathrm{~cm}^{-1}\right), \mathrm{U}_{2}\left(\sim 572 \mathrm{~cm}^{-1}\right)$ and $\mathrm{U}_{3}\left(\sim 630 \mathrm{~cm}^{-1}\right)$. They have previously been observed and interpreted differently [3-5].

Raman theory states that if these three peaks were to represent a change in polarisability connected to the phonons of the compound, then they should still appear, either with a lower or higher intensity, even if a different laser for the analysis is used. Hence, the characterization was again carried out with the $532 \mathrm{~nm}$ green laser. Comparing the spectrum obtained from the 633 $\mathrm{nm}$ to that of the $532 \mathrm{~nm}$ laser, it can be observed that the peaks appear at the same position which confirms the origin as Raman signals for these $\mathrm{UO}_{2}$ lines.

The obtained results for $\mathrm{PuO}_{2}$ are similar with those previously observed after irradiating $\mathrm{UO}_{2}$. Fig. 4(b) illustrates that the peaks remain at the same positions with different lasers and hence this also argues for a Raman origin of these lines in $\mathrm{PuO}_{2}$. 

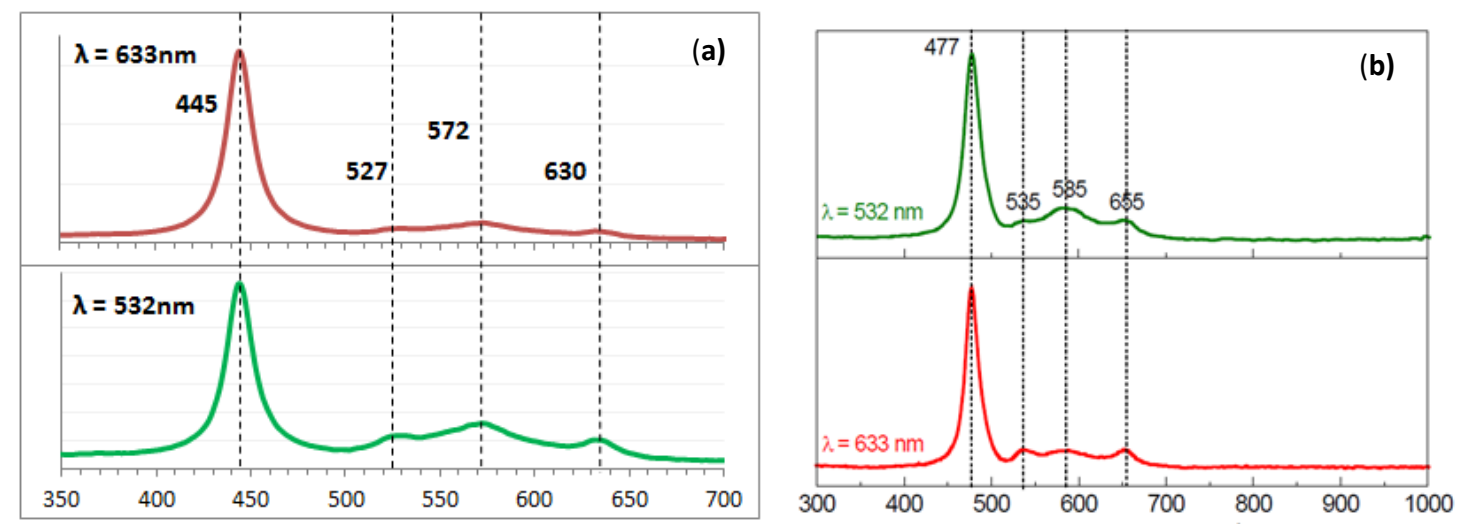

Fig. 4. Raman spectra of (a) $\mathrm{UO}_{2}$ (b) $\mathrm{PuO}_{2}$ when characterized using the $633 \mathrm{~nm}$ red \& $532 \mathrm{~nm}$ green laser

\section{$\underline{\mathrm{ThO}_{2}}$ Spectra}

The Raman spectrum of $\mathrm{ThO}_{2}$ (Fig. 5) shows that the $633 \mathrm{~nm}$ laser reveals the presence of four additional irradiation-induced peaks at $514 \mathrm{~cm}^{-1}, 539 \mathrm{~cm}^{-1}, 590 \mathrm{~cm}^{-1}$ and $622 \mathrm{~cm}^{-1}$. However, the subsequent characterization with the $532 \mathrm{~nm}$ laser shows that they fall to different positions, and moreover with different intensities. These results help to state that the peaks in $\mathrm{ThO}_{2}$ do not correlate to Raman scattering.

A luminescence mechanism can better explain the presence of these peaks. The energy of the photons from the laser is just sufficient to cause an absorption/emission process between different electronic energy levels in the material. The photons resulting from this process will be detected and appear as additional peaks on the Raman spectrum.

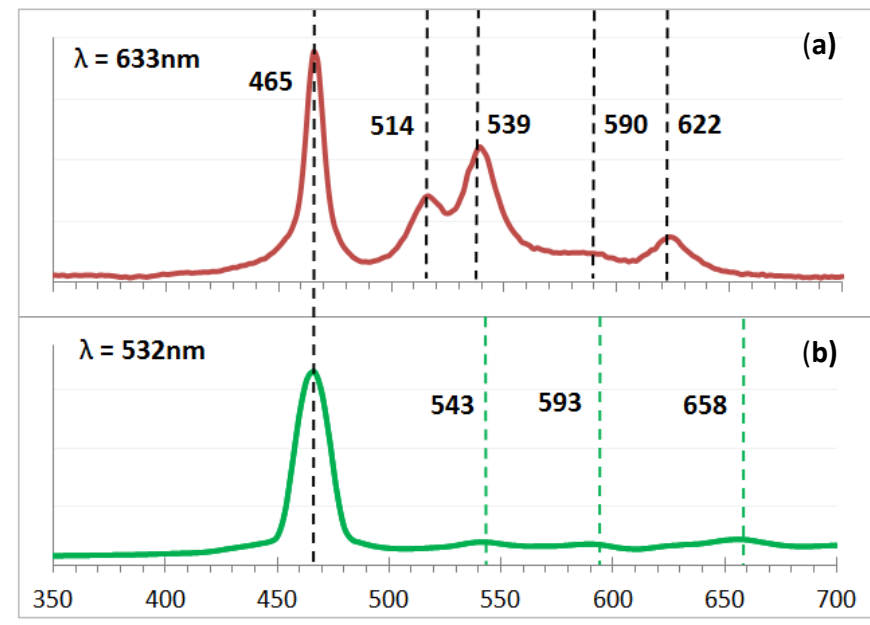

Fig. 5. Raman spectra of $\mathrm{ThO}_{2}$ with $633 \mathrm{~nm}$ and $532 \mathrm{~nm}$

\section{Discussions}

The results from the different experiments proved that $\mathrm{ThO}_{2}$ behaves differently under irradiation and this can be translated as an occurrence of a different mechanism of irradiation defects compared to $\mathrm{UO}_{2}$ and $\mathrm{PuO}_{2}$. Our study was mainly focused on the effect of electronic energy; therefore the stoichiometric nature of these compounds needs to be taken into account so as to propose a suitable mechanism of irradiation damage model in each case.

In $\mathrm{UO}_{2}, \mathrm{U}(\mathrm{III})$ and $\mathrm{U}(\mathrm{V})$ oxidation states can exist as well as the regular U(IV) [21]; while Pu(III) can also be formed in addition to $\mathrm{Pu}(\mathrm{IV})$ in $\mathrm{PuO}_{2}$ [22]. Thorium tends to form stoichiometric oxides and the most stable oxidation state remains +4 [23]. Hence based on the stoichiometry difference of these actinides, the following possible irradiation defects models are proposed.

\subsection{Mechanisms of Irradiation defects in $\mathrm{ThO}_{2}$}

According the Bethe stopping power theory, around $11.55 \mathrm{keV}$ is transferred to one electron during $21 \mathrm{MeV}$ helium beam irradiation conditions. This energy is largely enough to enhance electronic defects, causing the excitation of an electron from the valence band up to the conduction band. Considering the stoichiometry nature of $\mathrm{ThO}_{2}$, the electron will tend to decay back and recombine with its associated hole. This decay evidences the existence of a radiative channel which is consistent with the Ion-Beam Induced luminescence signals. This process does not have any effect on the normal lattice vibrations, thereby explaining the absence of Raman signals. On the other hand, the electrons are also involved in the formation of colored defects associated with the blue color in the irradiated $\mathrm{ThO}_{2}$ [24-25]. 


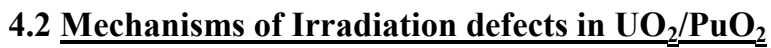

A unique mechanism could be held responsible for the irradiation defects in $\mathrm{UO}_{2}$ and $\mathrm{PuO}_{2}$. The initial stage can be described as similar to that of $\mathrm{ThO}_{2}$ whereby electronic defects are involved. However, absence of the luminescence signals proves that no such radiative channel exists in these oxides. This can be correlated with the nature of these compounds since once in the conduction band and instead of trying to decay to retain stoichiometry, the electrons will tend to interact with the atoms in the lattice thereby causing an eventual change in the polarization of the phonons. This process is better defined as an electronphonon coupling due to polarons [26, 27, 28-29] and will limit the light emission characteristics of $\mathrm{UO}_{2}$. Moreover the similarity of the Raman triplet in $\mathrm{UO}_{2}$ and $\mathrm{PuO}_{2}$ sustains an intrinsic origin, due to the fluorine structure.

\section{Conclusion}

To better define the mechanism of irradiation defects in different actinides due to the effect of alpha-irradiation, $\mathrm{UO}_{2}$ and $\mathrm{ThO}_{2}$ were externally irradiated with helium beam using a Cyclotron facility. The pellets were then characterized using luminescence, visual and Raman methods. On the other hand the $\mathrm{PuO}_{2}$, being too radioactive, had to be manipulated in a glove box and it was only possible to perform visual and Raman analyses.

This study shows that although $\mathrm{ThO}_{2}$ shares similar characteristics as $\mathrm{UO}_{2}$ and $\mathrm{PuO}_{2}$, its mechanism of defect creation is totally different. Obtained results for the initially white $\mathrm{ThO}_{2}$ showed that a blue color appeared in the irradiated areas and also the presence of luminescence signals during irradiation, while no new peaks were observed in its Raman spectrum. On the other hand, a Raman signature were observed during the post-mortem measurements of $\mathrm{UO}_{2}$ and $\mathrm{PuO}_{2}$, however no color change took place and the experimental set-up gave no indications of any luminescence signals. The results showed the existence of a radiative channel involving electronic defects in $\mathrm{ThO}_{2}$ while in $\mathrm{UO}_{2}$ and $\mathrm{PuO}_{2}$ the presence of a coupling between electronic defects and phonons was confirmed due to the Raman signature.

\section{References}

[1] H. Palancher, N. Wieschalla, P. Martin, R. Tucoulou, C. Sabathier, W. Petry, J.-F. Berar, C. Valot and S. Dubois, J Nucl. Mat. 385 (2009 449-455).

[2] I.L F. Ray, H. Thiele and Hj. Matzke, J Nucl Mater. 188 (1992) 90-95.

[3] G. Guimbretière, L. Desgranges, A. Canizarès, G. Carlot, R. Caraballo, C. Jégou, F. Duval, N. Raimboux, M. R. Ammar and P. Simon, Appl. Phys. Lett. 100 (2012) 251914.

[4] L. Desgranges, G. Guimbretière, P. Simon, F. Duval, A. Canizarès, R. Omnee, C. Jégou and R. Caraballo, Nucl. Instr. Methods B 327 (2014) 74-77.

[5] G.Guimbretière, L. Desgranges, A. Canizares, R. Caraballo, F. Duval, N. Raimboux, R. Omnée, MR Ammar, C. Jégou, P. Simon, Appl. Phys.Lett 103 (2013) 041904.

[6] T. Wiss, Hj. Matzke, C.Trautmann, M. Toulemonde, and S. Klaumünzer, J. Nucl Mater. 122 (1997) 583-588.

[7] R.T. Williams and K.S. Song, J. Phy. Chem. 51 (1990) 679-716.

[8] S. Watanabe,W.E.F. Ayta, J.R.B. Paiao, G.M. Ferraz, T.M.B. Farias and N.F. Cano, J. Phys. D: Appl. Phys. 41 (2008) 105401.

[9] J.M. Bodine, F.B. Theiss, Phys. Rev. 98 (1955) 1532.

[10] O.A. Weinreich, W.E. Danforth, Phys. Rev. 88 (1952) 953.

[11] R.C. Linares, J. Phys. Chem. Solids 28 (1967) 1285.

[12] J.L. Bates, Techn. Rep. BNWL-457, Contract At (45-1)-1830 (1967) 16.

[13] T.R. Griffiths, J. Dixon, Inorganica Chimica Acta 300-302 (2000) 305-313.

[14] Dolling, Cowley, Woods, Can. J. Phs. 43, 1397 (1965).

[15] Cowley, Dolling, Phys. Rev. 167, 464 (1968).

[16] L. Desgranges, G. Baldinozzi, P. Simon, G. Guimbretière and A. Canizarès, J. Raman Spectrosc. 43 (2012) 455-458.

[17] R. Böhler, A. Quainic, L. Capriottic, P. Çakırd, , O. Beneša, , K. Boboridisa, , A. Guiota, L. Luzzic, R.J.M. Koningsa, and D. Manara, J. Alloys and Compounds. 616 (2014) 5-13.

[18] T. Livneh, E. Sterer. Phys. Rev. B. 73 (2006) 085118.

[19] C. Jégou, R. Caraballo, S. Peuget, D. Roudil, L. Desgranges and M. Magnin, J. Nucl Mater. 405 (2010) $235-243$.

[20] M.J. Sarsfield, R.J. Taylor, C. Puxley and H.M. Steele, J. Nucl Mater. 427 (2012) 333-342.

[21] A. Jackson, A. D. Murray, J. H. Harding and C. R. A. Catlow, Phil. Mag A. 53 (1986) 27-50.

[22] S. D. Conradson, B. D. Begg ,D. L. Clark, C. D. Auwer, F. J. Espinosa-Faller ,P. L. Gordon ,N.J. Hess, R. Hess , D. W. Keogh , L. A. Morales , M. P. Neu ,W. Runde, C. D. Tait, D. K. Veirs and P. M. Villella, Inorg. Chem. 42 (2003) 3715-3717.

[23] Kelly, P.J. \& Brooks, M.S.S. J. Chem. Soc. 1987. 83: 1189-1203. 
[24] B.G. Childs, P.J. Harvey and J.B. Hallett, J. Am. Chem. Soc. 53 (1970) 431.

[25] V. I. Neeley, J.B. Gruber and W. J. Gray, Phy. Rev. 158 (1967) 809.

[26] J.M. Casado, J.H. Harding and G.J. Hyland, J. Phys.: Condens. Matter 6 (1994) 4685.

[27] C. Ronchi, M. Sheindlin, M. Musella and G. J. Hyland, J. Appl. Phys. 85 (1999) 776.

[28] Y.Q. An, A.J. Taylor, T. Durakiewicz, and G.Rodriguez, J. Phys. Conf. 273 (2011) 012144.

[29] P. Ruello, G. Petot-Ervas, C. Petot and L.Desgranges, J. Am. Ceram. Soc. 88 (2005) 604. 